\title{
Solutions de similitude d'un jeu différentiel stochastique
}

\author{
Mario Lefebvre \\ Département de mathématiques et de génie industriel \\ École Polytechnique de Montréal \\ C.P. 6079, Succursale Centre-ville \\ Montréal, Québec H3C 3A7 \\ Canada \\ mlefebvre@polymtl.ca
}

RÉSUMÉ. On considère un processus stochastique commandé bidimensionnel défini par un ensemble d'équations différentielles stochastiques. Contrairement à la formulation la plus fréquente, les variables de commande apparaissent dans les variances infinitésimales du processus, plutôt que dans les moyennes infinitésimales. Le jeu différentiel prend fin lorsque les deux processus sont égaux ou que leur différence est égale à une constante donnée. Des solutions explicites à des problèmes particuliers sont obtenues en utilisant la méthode des similitudes pour résoudre l'équation aux dérivées partielles appropriée.

ABSTRACT. A two-dimensional controlled stochastic process defined by a set of stochastic differential equations is considered. Contrary to the most frequent formulation, the control variables appear only in the infinitesimal variances of the process, rather than in the infinitesimal means. The differential game ends the first time the two controlled processes are equal or their difference is equal to a given constant. Explicit solutions to particular problems are obtained by making use of the method of similarity solutions to solve the appropriate partial differential equation.

MOTS-CLÉS : théorie des jeux, mouvement brownien bidimensionnel, programmation dynamique.

KEYWORDS : game theory, two-dimensional Brownian motion, dynamic programming. 


\section{Introduction}

Soit $X(t)$ un processus de diffusion commandé unidimensionnel défini par l'équation différentielle stochastique

$$
d X(t)=f(X(t)) d t+\{|u(t)| v(X(t))\}^{1 / 2} d W(t)
$$

où $f(x)$ et $v(x)$ sont des fonctions positives si $x>d_{1}$, et $W(t)$ est un mouvement brownien standard. Dans [2], l'auteur a considéré le problème de minimiser l'espérance mathématique de la fonction de coût

$$
J(x)=\int_{0}^{\tau(x)}\left[\frac{1}{2} q(X(t)) u^{2}(t)+\lambda\right] d t+K[X(\tau)]
$$

où $\lambda$ est une constante réelle,

$$
\tau(x)=\inf \left\{t \geq 0: X(t) \in\left\{d_{1}, d_{2}\right\} \mid X(0)=x \in\left[d_{1}, d_{2}\right]\right\}
$$

et le coût final est donné par

$$
K[X(\tau)]=\left\{\begin{array}{cc}
0 & \text { si } X(\tau)=d_{1} \\
K_{0} & \text { si } X(\tau)=d_{2}
\end{array}\right.
$$

où $K_{0}$ est une constante positive.

Dans le cas où la constante $\lambda$ est positive, l'objectif est de faire en sorte que le processus commandé $X(t)$ quitte l'intervalle $\left[d_{1}, d_{2}\right]$ aussitôt que possible, tandis que, lorsque $\lambda<0$, on cherche à maximiser le temps de survie dans l'intervalle $\left[d_{1}, d_{2}\right]$ (en tenant compte des coûts de commande quadratiques dans les deux cas, et également lorsque $\lambda=0$ ). Ce type de problème est une modification des problèmes que Whittle (voir [3] ou [4]) a appelés $L Q G$ homing.

Dans le présent article, plutôt que de considérer un problème de commande optimale stochastique en une dimension, nous voulons trouver la solution d'un jeu différentiel stochastique. On définit le processus de diffusion commandé bidimensionnel $\left(X_{1}(t), X_{2}(t)\right)$ par les équations différentielles stochastiques

$$
d X_{i}(t)=a_{i}\left(X_{i}(t)\right) d t+\left\{\left|u_{i}(t)\right| v_{i}\left(X_{i}(t)\right)\right\}^{1 / 2} d W_{i}(t)
$$

pour $i=1,2$, où $W_{1}(t)$ et $W_{2}(t)$ sont des mouvements browniens standards indépendants. La fonction $a_{i}(\cdot)$ est maintenant une fonction réelle, donc pas nécessairement positive.

Remarque. Il est à noter que c'est la variance infinitésimale de $\left(X_{1}(t), X_{2}(t)\right)$ qui dépend de la commande, plutôt que sa moyenne infinitésimale, comme dans la formulation classique. Une raison importante pour laquelle les auteurs supposent généralement que la variable de commande agit uniquement sur la partie déterministe de l'équation différentielle stochastique est que cela conduit à une solution plus facile à calculer. Cependant, il est logique de supposer que le fait d'exercer une certaine commande sur un processus augmente ou diminue sa variance infinitésimale. Ainsi, dans la formulation ci-dessus, si $u_{i}(t) \equiv 0$, alors le processus $\left(X_{1}(t), X_{2}(t)\right)$ est purement déterministe, tandis que dans le cas classique, le fait de choisir $u_{i}(t) \equiv 0$ implique que $\left(X_{1}(t), X_{2}(t)\right)$ demeure un processus de diffusion bidimensionnel. L'équation (5) peut être plus réaliste dans plusieurs 
applications. Naturellement, on pourrait aussi considérer le cas où les variables de commande $u_{i}(t)$ influencent à la fois la moyenne et la variance infinitésimales du processus.

On désire trouver les valeurs des variables $u_{i}(t)$ qui optimisent l'espérance mathématique de la fonction de coût

$$
J\left(x_{1}, x_{2}\right)=\int_{0}^{T}\left[\lambda+u_{1}^{2}(t)-u_{2}^{2}(t)\right] d t+K\left[X_{1}(T), X_{2}(T)\right]
$$

où, comme dans le cas unidimensionnel, $\lambda$ est une constante, et le coût final est

$$
K\left[X_{1}(T), X_{2}(T)\right]= \begin{cases}0 & \text { si } X_{1}(T)-X_{2}(T)=0 \\ k & \text { si } X_{1}(T)-X_{2}(T)=d\end{cases}
$$

où $k$ est une constante positive. De plus, le temps final $T=T\left(x_{1}, x_{2}\right)$ est une variable aléatoire :

$$
T\left(x_{1}, x_{2}\right)=\inf \left\{t>0: X_{1}(t)-X_{2}(t)=0 \text { ou } d \mid X_{i}(0)=x_{i}\right\}
$$

où l'on suppose que $0<x_{1}-x_{2}<d$. C'est-à-dire que le jeu prend fin lorsque l'un ou l'autre de deux événements se produit pour la première fois : les deux processus sont égaux, ou bien leur différence est égale à la constante $d$.

Dans un jeu différentiel, il y a deux optimiseurs; le premier (à l'aide de la variable $u_{1}(t)$ ) veut minimiser l'espérance mathématique de $J$, tandis que le deuxième (à l'aide de $\left.u_{2}(t)\right)$ essaie de la maximiser. Lorsque le paramètre $\lambda$ est positif, le premier optimiseur désire que le processus $X_{1}(t)$ rejoigne le processus $X_{2}(t)$ aussitôt que possible, tandis que le deuxième optimiseur veut que $X_{2}(t)$ s'échappe le plus longtemps possible. Les deux optimiseurs doivent bien sûr tenir compte des coûts de commande quadratiques.

Dans le cas où $\lambda<0$, le premier optimiseur doit faire un compromis entre la récompense donnée pour le temps de survie du processus dans l'intervalle $(0, d)$, et les coûts de commande quadratiques et le coût final $k$ imposé si $X_{1}(T)-X_{2}(T)=d$. De même, le deuxième optimiseur doit prendre en considération le fait que la récompense donnée pour le temps que $X_{1}(t)-X_{2}(t)$ passe dans l'intervalle $(0, d)$ peut être supérieure au coût final $k$.

Enfin, lorsque $\lambda=0$, le temps que le processus commandé passe dans la région de continuation n'est ni pénalisé ni récompensé directement. Cependant, si $u_{i}(t) \neq 0$, il est avantageux pour les deux optimiseurs que le jeu se termine le plus rapidement possible.

Dans la prochaine section, les solutions optimales $u_{i}^{*}$ de notre problème seront exprimées en fonction de la fonction de valeur. Dans la section 3, des valeurs optimales de $u_{1}$ et $u_{2}$ seront calculées explicitement dans deux problèmes importants en utilisant un cas particulier de la méthode des similitudes pour résoudre l'équation aux dérivées partielles appropriée. Enfin, nous terminerons ce travail par quelques remarques.

\section{Commandes optimales}

Pour résoudre notre problème, nous considérons

$$
\begin{array}{ccc}
F\left(x_{1}, x_{2}\right)= & \min _{\substack{u_{1}(t) \\
0 \leq t \leq T}} \quad \max _{u_{2}(t)} E\left[J\left(x_{1}, x_{2}\right)\right] \\
& 0 \leq t \leq T
\end{array}
$$


On suppose que la fonction de valeur $F$, qui donne la valeur optimale de l'espérance mathématique de la fonction de coût $J\left(x_{1}, x_{2}\right)$, existe et est deux fois dérivable par rapport à $x_{1}$ et $x_{2}$. On peut facilement montrer (en généralisant les résultats dans [3]) que $F$ satisfait à l'équation de programmation dynamique

$$
0=\min _{u_{1}} \max _{u_{2}}\left[\lambda+u_{1}^{2}-u_{2}^{2}+\sum_{i=1}^{2}\left(a_{i} F_{x_{i}}+\frac{1}{2} v_{i}\left|u_{i}\right| F_{x_{i} x_{i}}\right)\right]
$$

où les variables $u_{i}, a_{i}$ et $v_{i}$ sont les valeurs des fonctions correspondantes évaluées à l'instant initial $0: u_{i}=u_{i}(0), a_{i}=a_{i}\left(X_{i}(0)\right)$ et $v_{i}=v_{i}\left(X_{i}(0)\right)$, pour $i=1,2$. De plus, $F_{x_{i}}=\frac{\partial F}{\partial x_{i}}$ et $F_{x_{i} x_{i}}=\frac{\partial^{2} F}{\partial x_{i}^{2}}$.

On peut immédiatement énoncer le résultat suivant.

Lemme 2.1 Si $F_{x_{1} x_{1}}$ est non négatif, alors $u_{1}^{*}=0$. De même, dans le cas où $F_{x_{2} x_{2}} \leq 0$, on $a: u_{2}^{*}=0$.

Les solutions ne sont pas triviales lorsque $F_{x_{1} x_{1}}<0$ ou $F_{x_{2} x_{2}}>0$.

Proposition 2.1 Si l'on suppose que la fonction $F$ existe et est deux fois dérivable par rapport à $x_{1}$ et $x_{2}$, alors les commandes optimales sont données par

$$
u_{1}^{*}=-\frac{1}{4} v_{1} F_{x_{1} x_{1}} \quad \text { si } F_{x_{1} x_{1}}<0
$$

et

$$
u_{2}^{*}=\frac{1}{4} v_{2} F_{x_{2} x_{2}} \quad \text { si } F_{x_{2} x_{2}}>0
$$

Preuve Il suffit de remarquer que, puisque $u_{i}$ n'apparaît qu' au carré ou en valeur absolue dans l'équation (10), on peut supposer que $u_{i} \geq 0$. Le résultat découle alors immédiatement de (10), par dérivation.

Il est aisé d'obtenir les solutions optimales en fonction de $F$. Cependant, il n'est pas aussi facile de trouver des solutions explicites pour des problèmes particuliers importants.

Étant donné que la fonction de coût final ne dépend que de la différence entre les variables, nous allons supposer que

$$
F\left(x_{1}, x_{2}\right)=G(z)
$$

où $z=x_{1}-x_{2}$. Notons que cela implique que

$$
F_{x_{1} x_{1}}\left(x_{1}, x_{2}\right)=F_{x_{2} x_{2}}\left(x_{1}, x_{2}\right)=G^{\prime \prime}(z)
$$

Par conséquent, on ne peut pas avoir à la fois $F_{x_{1} x_{1}}<0$ et $F_{x_{2} x_{2}}>0$. Il s'ensuit que l'une des deux commandes optimales sera nulle, tandis que l'autre sera strictement positive. Dans le cas où $F_{x_{1} x_{1}}$ et $F_{x_{2} x_{2}}$ sont négatifs, en substituant (11) dans l'équation de programmation dynamique, on trouve que l'on doit résoudre l'équation aux dérivées partielles

$$
\lambda-\frac{1}{16} v_{1}^{2} F_{x_{1} x_{1}}^{2}+\sum_{i=1}^{2} a_{i} F_{x_{i}}=0
$$


Les conditions limites sont

$$
F\left(x_{1}, x_{2}\right)= \begin{cases}0 & \text { si } x_{1}-x_{2}=0 \\ k & \text { si } x_{1}-x_{2}=d\end{cases}
$$

où $d>0$. Sous l'hypothèse $F\left(x_{1}, x_{2}\right)=G(z)$, cette équation est alors transformée en l'équation différentielle ordinaire (non linéaire)

$$
\lambda-\frac{v_{1}^{2}}{16}\left[G^{\prime \prime}(z)\right]^{2}+\left(a_{1}-a_{2}\right) G^{\prime}(z)=0
$$

et les conditions limites deviennent $G(0)=0$ et $G(d)=k$.

Notons que pour que cette hypothèse puisse être valable, il faut au moins que les coefficients des dérivées $G^{\prime}(z)$ et $G^{\prime \prime}(z)$ s'expriment aussi en fonction de la variable de similitude $z$. Cela est bien sûr possible, en particulier, si $v_{i}$ et $a_{i}$ sont des constantes, pour $i=1,2$. Dans la prochaine section, nous verrons que cette technique de résolution d'une équation aux dérivées partielles nous permet effectivement d'obtenir des solutions explicites dans deux problèmes importants.

Dans le cas où les dérivées partielles $F_{x_{i} x_{i}}$ sont positives, pour $i=1$, 2 , on trouve que l'équation à résoudre est

$$
\lambda+\frac{v_{2}^{2}}{16}\left[G^{\prime \prime}(z)\right]^{2}+\left(a_{1}-a_{2}\right) G^{\prime}(z)=0
$$

(et les conditions limites sont les mêmes).

Remarque. Nous allons trouver des solutions exactes à nos problèmes en utilisant la méthode des similitudes. Théoriquement, il pourrait exister d'autres solutions que celles que nous allons obtenir, puisque nous ne considérons pas le problème de l'unicité des solutions.

\section{Solutions explicites}

Cas I. Supposons d'abord que $a_{i}\left(X_{i}(t)\right) \equiv 0$ et $v_{i}\left(X_{i}(t)\right) \equiv 1$, de sorte que

$$
d X_{i}(t)=\left\{\left|u_{i}(t)\right|\right\}^{1 / 2} d W_{i}(t)
$$

En prenant $u_{i}(t) \equiv 0$, pour $i=1,2$, on aurait : $X_{i}(t)=x_{i}$ pour tout $t \geq 0$. Comme on a supposé que $0<x_{1}-x_{2}<d$, on peut conclure que $T\left(x_{1}, x_{2}\right)=\infty$ et que

$$
E\left[J\left(x_{1}, x_{2}\right)\right]=\lambda E\left[T\left(x_{1}, x_{2}\right)\right]=\lambda \times \infty
$$

Cela pourrait être la solution optimale (triviale) si les deux optimiseurs cherchaient à maximiser (si $\lambda>0$ ) ou à minimiser (si $\lambda<0) E\left[J\left(x_{1}, x_{2}\right)\right]$. Cependant, ici les deux optimiseurs ont des objectifs opposés et la solution optimale n'est pas (en général) $u_{i}(t) \equiv 0$, pour $i=1,2$.

Remarque. Nous avons affirmé que les cas particuliers que nous considérons sont des problèmes importants. Cette affirmation est justifiée par le fait que si $u_{i}(t) \equiv 1$, alors $\left(X_{1}(t), X_{2}(t)\right)$ est un mouvement brownien standard bidimensionnel (voir [1], par exemple). De façon plus générale, si $u_{i}(t)$ est une constante (non nulle) quelconque, alors $\left(X_{1}(t), X_{2}(t)\right)$ est un processus de Wiener (bidimensionnel). Il s'agit du processus de diffusion le plus important pour les applications. 
a) Si l'on suppose que $G^{\prime \prime}(z)$ est strictement négatif, on doit résoudre

$$
\lambda-\frac{1}{16}\left[G^{\prime \prime}(z)\right]^{2}=0
$$

de sorte que

$$
G^{\prime \prime}(z)=-4 \sqrt{\lambda}
$$

Théoriquement, le paramètre $\lambda$ peut prendre n'importe quelle valeur réelle. Cependant, ici on voit que l'on doit choisir $\lambda$ plus grand que zéro. C'est-à-dire que l'on doit pénaliser le temps que le processus passe dans l'intervalle $(0, d)$ avant de frapper l'une ou l'autre des frontières.

De là, la commande optimale $u_{1}^{*}$ est donnée par

$$
u_{1}^{*}=-\frac{1}{4} G^{\prime \prime}(z)=\sqrt{\lambda}
$$

Ainsi, la commande optimale est constante dans ce cas.

On peut aussi calculer explicitement la fonction de valeur $F\left(x_{1}, x_{2}\right)$. On trouve que

$$
F\left(x_{1}, x_{2}\right)=-2 \sqrt{\lambda}\left(x_{1}-x_{2}\right)^{2}+\left(k+2 \sqrt{\lambda} d^{2}\right) \frac{\left(x_{1}-x_{2}\right)}{d}
$$

pour $x_{1}-x_{2}$ dans l'intervalle $[0, d]$.

b) Lorsque $G^{\prime \prime}(z)$ est strictement positif, on a :

$$
\lambda+\frac{1}{16}\left[G^{\prime \prime}(z)\right]^{2}=0 \quad \Longrightarrow \quad G^{\prime \prime}(z)=4 \sqrt{-\lambda}
$$

où l'on doit maintenant supposer que $\lambda$ est négatif. Cela signifie que l'on doit donner une récompense pour le temps que le processus passe dans la région de continuation $(0, d)$ avant de frapper l'une ou l'autre des frontières.

On déduit alors que la commande optimale $u_{2}^{*}$ est

$$
u_{2}^{*}=\frac{1}{4} G^{\prime \prime}(z)=\sqrt{-\lambda}
$$

et

$$
F\left(x_{1}, x_{2}\right)=2 \sqrt{-\lambda}\left(x_{1}-x_{2}\right)^{2}+\left(k-2 \sqrt{-\lambda} d^{2}\right) \frac{\left(x_{1}-x_{2}\right)}{d}
$$

pour $0 \leq x_{1}-x_{2} \leq d$.

Remarques. i) Lorsque $a_{i}\left(X_{i}(t)\right) \equiv 0$ et $v_{i}\left(X_{i}(t)\right) \equiv 1$, l'équation aux dérivées partielles (15) devient :

$$
\lambda-\frac{1}{16} F_{x_{1} x_{1}}^{2}=0 \quad \text { ou } \quad \lambda+\frac{1}{16} F_{x_{2} x_{2}}^{2}=0
$$

Cette équation peut en fait être résolue sans utiliser la méthode des similitudes.

ii) Il est logique que $u_{2}^{*}$ soit nul lorsque le paramètre $\lambda$ est positif, car le deuxième optimiseur cherche à maximiser la valeur de l'espérance mathématique de la fonction de coût. Étant donné que le temps passé dans l'intervalle $(0, d)$ est pénalisé dans ce cas, il est préférable que le deuxième optimiseur fasse en sorte que le processus $X_{2}(t)$ ne bouge pas de sa position initiale $x_{2}$. Inversement, si $\lambda$ est négatif, alors le premier optimiseur devrait essayer de prolonger le jeu le plus longtemps possible, d'où le choix de $u_{1}^{*}$ égal à 
0 , afin que $X_{1}(t)$ ne bouge pas d'où il se trouve au départ. Notons qu'ici le fait d'utiliser une valeur non nulle de $u_{i}(t)$ n'a pour effet que d'augmenter la variance de $X_{i}(t)$, augmentant en même temps la probabilité que le jeu se termine rapidement. En effet, si, par exemple, $u_{i}(t) \equiv k_{i} \geq 0$, pour $i=1,2$, alors $X_{i}(t)$ est un processus de Wiener de paramètres infinitésimaux 0 et $k_{i}$. De plus, $X_{1}(t)-X_{2}(t)$ est aussi un processus de Wiener, de paramètres infinitésimaux 0 et $k_{1}+k_{2}$. Or, on trouve que l'espérance mathématique de la variable aléatoire $T\left(x_{1}, x_{2}\right)$ dans ce cas est donnée par

$$
E\left[T\left(x_{1}, x_{2}\right)\right]=\frac{2\left(x_{1}-x_{2}\right)}{k_{1}+k_{2}}\left[d-\left(x_{1}-x_{2}\right)\right] \quad \text { pour } 0 \leq x_{1}-x_{2} \leq d
$$

Ainsi, on peut effectivement affirmer que plus $k_{1}$ et $k_{2}$ sont grands, plus le temps moyen que dure le jeu est court.

iii) On peut se demander pourquoi les commandes optimales ne dépendent pas de la constante $k$. La raison est que, dans ce cas particulier, les moyennes infinitésimales des processus $X_{i}(t)$ sont nulles. Supposons, comme dans la remarque précédente, que $u_{i}(t) \equiv$ $k_{i} \geq 0$, pour $i=1,2$. On peut montrer qu'on a alors :

$$
P\left[X_{1}(T)-X_{2}(T)=d\right]=\frac{x_{1}-x_{2}}{d} \quad \text { pour } 0 \leq x_{1}-x_{2} \leq d
$$

On voit que la probabilité que le jeu se termine avec $X_{1}(t)-X_{2}(t)=d$ ne dépend que de la valeur de $X_{1}(0)$ et $X_{2}(0)$. Il ne sert donc à rien d'essayer d'augmenter (ou de diminuer) cette probabilité en utilisant des valeurs plus ou moins grandes de $u_{i}(t)$.

Cas II. Nous pouvons généraliser le cas précédent en supposant maintenant que $a_{i}\left(X_{i}(t)\right)$ $\equiv \mu_{i}$ et $v_{i}\left(X_{i}(t)\right) \equiv \sigma_{i}^{2}(>0)$. Si $u_{i}(t) \equiv 1$, alors $\left(X_{1}(t), X_{2}(t)\right)$ est un processus de Wiener bidimensionnel dont les paramètres infinitésimaux sont $\mu_{i}$ et $\sigma_{i}^{2}$, pour $i=1,2$. Par souci de simplicité, nous ne considérons que le cas particulier suivant :

$$
\sigma_{1}^{2}=\sigma_{2}^{2}=1, \quad \mu_{1}=0, \quad \mu_{2}=1, \quad d=1
$$

a) Si l'on suppose que $G^{\prime \prime}(z)<0$, l'équation (17) devient alors

$$
\lambda-\frac{1}{16}\left[G^{\prime \prime}(z)\right]^{2}-G^{\prime}(z)=0
$$

Cette équation différentielle ordinaire non linéaire possède la solution

$$
G(z)=-\frac{4}{3} z^{3}-4 c_{1} z^{2}+\left(\lambda-4 c_{1}^{2}\right) z+c_{2}
$$

où $c_{1}$ et $c_{2}$ sont des constantes. La condition limite $G(0)=0$ implique que $c_{2}=0 ;$ on déduit alors de l'autre condition, $G(1)=k$, que

$$
c_{1}=-\frac{1}{2} \pm\left[-\frac{1}{12}+\frac{\lambda+k}{4}\right]^{1 / 2}
$$

Notons que l'on doit avoir :

$$
\lambda+k-\frac{1}{3} \geq 0 \quad \Longleftrightarrow \quad \lambda \geq \frac{1}{3}-k
$$


De plus, on a :

$$
G^{\prime \prime}(z)=-8 z-8 c_{1}<0 \quad \forall z \in[0,1] \quad \Longrightarrow \quad c_{1}>0
$$

Il s'ensuit que l'on doit choisir le signe "+" dans l'équation (34) et que

$$
-\frac{1}{2}+\left[-\frac{1}{12}+\frac{\lambda+k}{4}\right]^{1 / 2}>0 \quad \Longrightarrow \quad \lambda>\frac{4}{3}-k
$$

Comme nous avons fait l'hypothèse que la constante $k$ est strictement positive, on peut conclure que le paramètre $\lambda$ peut maintenant être négatif, si $k>4 / 3$. On peut donc pénaliser, jusqu'à un certain point, le temps que le processus passe dans l'intervalle $(0,1)$. D'un autre côté, on peut affirmer que le coût $k$ imposé lorsque le processus $\left(X_{1}(t), X_{2}(t)\right)$ est absorbé en $X_{1}(t)-X_{2}(t)=d$ doit être supérieur à $4 / 3$ moins le paramètre $\lambda$.

La commande optimale $u_{1}^{*}$ est donnée par

$$
u_{1}^{*}=8\left(x_{1}-x_{2}+c_{1}\right)
$$

ce qui est effectivement positif pour $x_{1}-x_{2} \in[0,1]$, car $c_{1}$ est positif. Donc, la commande optimale est maintenant linéaire en $x_{1}-x_{2}$ plutôt qu'une constante comme dans le cas I.

b) L'équation à résoudre si $G^{\prime \prime}(z)>0$ est (voir (18))

$$
\lambda+\frac{1}{16}\left[G^{\prime \prime}(z)\right]^{2}-G^{\prime}(z)=0
$$

On trouve que

$$
G(z)=\frac{4}{3} z^{3}+4 c_{1} z^{2}+\left(\lambda+4 c_{1}^{2}\right) z+c_{2}
$$

Comme ci-dessus, on déduit de la condition limite $G(0)=0$ que la constante $c_{2}$ est nulle, et alors $G(1)=k$ implique que

$$
c_{1}=-\frac{1}{2} \pm\left[-\frac{1}{12}+\frac{-\lambda+k}{4}\right]^{1 / 2}
$$

Pour que $G^{\prime \prime}(z)$ soit effectivement positif pour tout $z \in[0,1]$, il faut que la constante $c_{1}$ soit positive. Il s'ensuit que l'on doit choisir le signe “+” dans l'équation ci-dessus.

De plus, dans ce cas, on trouve que la condition

$$
\lambda<k-\frac{4}{3}
$$

doit être vérifiée pour que la solution obtenue soit valable. Donc ici le paramètre $\lambda$ peut être positif, si $k>4 / 3$.

Enfin, comme précédemment, la valeur optimale de la commande $u_{2}$ est

$$
u_{2}^{*}=8\left(x_{1}-x_{2}+c_{1}\right)
$$

Remarque. Si l'on pose que $u_{i}(t) \equiv 0$, pour $i=1,2$, alors les processus $X_{1}(t)$ et $X_{2}(t)$ sont tels que

$$
d X_{1}(t)=0 \quad \Longrightarrow \quad X_{1}(t)=X_{1}(0)=x_{1}
$$


et

$$
d X_{2}(t)=d t \quad \Longrightarrow \quad X_{2}(t)=X_{2}(0)+t=x_{2}+t
$$

pour tout $t \geq 0$. On déduit de l'hypothèse $0<x_{1}-x_{2}<1$ que $T\left(x_{1}, x_{2}\right)=x_{1}-x_{2}$ et que $X_{1}(T)-X_{2}(T)=0$, de sorte que $K\left[X_{1}(T), X_{2}(T)\right]=0$. Il s'ensuit que

$$
J\left(x_{1}, x_{2}\right)=\lambda T\left(x_{1}, x_{2}\right)=\lambda\left(x_{1}-x_{2}\right)
$$

Puisque $T\left(x_{1}, x_{2}\right)$ devient une variable déterministe dans ce cas, on peut aussi écrire que $E\left[J\left(x_{1}, x_{2}\right)\right]=\lambda\left(x_{1}-x_{2}\right)$. On voit que cette solution n'est pas la solution optimale (voir (33)).

\section{Conclusion}

Nous avons obtenu des solutions analytiques d'un jeu différentiel stochastique. Les cas particuliers traités peuvent être considérés comme des processus de Wiener commandés. Il s'agit de processus qui apparaissent dans de très nombreuses applications. Le processus de Wiener est en fait le processus de diffusion de base.

Le jeu différentiel considéré prenait fin dès que la différence entre les deux processus commandés devenait nulle ou égale à une constante positive $d$. Comme suite de ce travail, on pourrait essayer de résoudre des problèmes pour lesquels, par exemple,

$$
K\left[X_{1}(T), X_{2}(T)\right]= \begin{cases}0 & \text { si } X_{1}(T)=X_{2}(T) \\ k & \text { si } X_{1}(T)=c X_{2}(T)\end{cases}
$$

avec

$$
T\left(x_{1}, x_{2}\right)=\inf \left\{t>0: X_{1}(t) / X_{2}(t)=1 \text { ou } c \mid X_{i}(0)=x_{i}\right\}
$$

où $1<x_{1} / x_{2}<c$. Ainsi, plutôt qu' une région du plan située entre deux droites parallèles, la région de continuation serait la partie du plan comprise entre deux droites qui se croisent à l'origine. On rechercherait alors des solutions de la forme

$$
F\left(x_{1}, x_{2}\right)=H\left(\frac{x_{1}}{x_{2}}\right)
$$

On pourrait aussi remplacer l'équation différentielle stochastique par

$$
d X_{i}(t)=a_{i}\left(X_{i}(t)\right) d t+\left\{u_{i}^{2}(t) v_{i}\left(X_{i}(t)\right)\right\}^{1 / 2} d W_{i}(t)
$$

et la fonction de coût par

$$
J\left(x_{1}, x_{2}\right)=\int_{0}^{T}\left[\lambda+u_{1}^{4}(t)-u_{2}^{4}(t)\right] d t+K\left[X_{1}(T), X_{2}(T)\right]
$$

Finalement, on pourrait essayer d'autres techniques, comme la méthode de séparation des variables, pour résoudre l'équation de programmation dynamique.

\section{Remerciements}

Cette recherche a été subventionnée par le Conseil de recherches en sciences naturelles et en génie du Canada. L'auteur remercie également les rapporteurs de cet article pour leurs commentaires constructifs. 


\section{Bibliographie}

[1] D.R. Cox, H.D. Miller, The Theory of Stochastic Processes, Methuen, London, 1965.

[2] M. LEFEB VRE, «A homing problem for diffusion processes with control-dependent variance », Annals of Applied Probability, vol. 14, $\mathrm{n}^{\circ}$ 2, p. 786-795, 2004.

[3] P. Whittle, Optimization over Time. Volume I : Dynamic Programming and Stochastic Control, Wiley, Chichester, 1982.

[4] P. Whittle, Risk-sensitive Optimal Control, Wiley, Chichester, 1990. 